\title{
EDITORIAL
}

\section{A new EM journal?}

\author{
Garth Dickinson, MD
}

A nother emergency medicine journal? There are scads already! Some arrive even if you don't subscribe. CAEP has already tried this. Remember the CAEP Review from the early '80s? It wasn't just the lousy name. Canada is too small; Canadian emerg docs are too busy to write. And what about the trees?

Canadians are a modest bunch and reasonably so. Our country is big on land but short on people. We're part of the G8, and rich, but we're hardly an economic dynamo. We don't have a long history like the Greeks or Italians. We never conquered half the world like the British or French, and our military might limits us to picking off the odd stray Spanish fishing trawler. We are polite and culturally sensitive. Step out of the country and try to find any evidence of intelligent life emanating from that big, pink smudge on the globe that separates the US from Santa. Only environmental catastrophes and Quebec separatists bring Canada international notice.

Of course, Canada is just a well kept secret. We continually top the United Nations' annual list of best countries in the world to live. Even without the benefit of the CRTC, radio stations around the world blast out the tunes of

Division of Emergency Medicine, University of Ottawa, presently on leave in Harare, Zimbabwe
Céline Dion, Bryan Adams, Alanis Morissette and Shania Twain - all naturally assumed to be American. That impossible, underfunded health care system that Canadian physicians love to hate is actually the envy of much of the rest of the world.

Pick up your favourite US emergency medicine journal and flip through the list of contributors. Quite a few Canadians, eh? Our emergency medicine scholars deserve more recognition than they receive. We are the second oldest established EM specialty in the world and one of the four founding members of the International Federation of Emergency Medicine. The Canadian contribution to the world literature in emergency medicine vastly exceeds our humble size.

But do we really need another EM journal? The truth is, of 3330 journals listed in Index Medicus, only 12 deal with emergency medicine and 2 of these are published in German. Of the 10 English-language journals, 3 are from the UK and 7 from the US. Three of these journals deal specifically with the subspecialty areas of disaster medicine and pediatric emergency medicine.

The Canadian health care system is unique, and, as we all know, emergency medicine is what keeps it going. EM has global generic challenges, but Canadian EM's specific strengths, problems and solutions are distinctly different from those of the rest of the world. Funding for our system is like no other. Does reading about the threats of managed care make your eyes glaze over? We don't

\section{... of $\mathbf{3 3 3 0}$ journals listed in Index Medicus, only 12 deal with emergency medicine ...}

have COBRAs stalking us in the ED, but we do have our own demons: the "dump" and "no beds" species, to name but two.

Try convincing a US emergency physician that quality assurance is about improving patient care and not about saving malpractice premiums. And we're not only different from Americans; try discussing the value of the CCFP(EM) with an Australian or a Scot. Our gentle multicultural mix of citizens rarely provides us the excuse for a thoracotomy, but flying home from anywhere can bring us in contact with just about any disease or condition known to man.

Canadian emergency physicians will keep publishing in the global press, helping to sell magazines and remedies for their advertisers. But it's time now to have the option of publishing in Canada and to share the Canadian EM experience with the world beyond. We need a Canadian journal of emergency medicine. 


\title{
ÉDITORIAL
}

\section{Un nouveau journal de médecine d'urgence?}

\author{
Garth Dickinson, MD
}

$\mathrm{U}$ n autre journal de médecine d'urgence? Il y en déjà des tonnes! Certains vous parviennent sans que vous y soyiez abonné. Vous souvenez-vous du CAEP Review au début des années 80 ? Il n'y avait pas que le titre nul. Le Canada est trop petit; les urgentologues canadiens sont trop occupés pour écrire.

Les Canadiens forment un peuple modeste et avec raison. Notre pays couvre un immense territoire mais les habitants sont peu nombreux. Nous faisons partie du G8 et nous sommes riches, mais nous sommes loins d'être une grande force économique. Notre histoire n'est pas aussi ancienne que celle des Grecs ou des Italiens. Nous n'avons jamais conquis la moitié du monde comme les Britanniques ou les Français et notre puissance militaire se limite à l'interception occasionnelle du chalutier espagnol égaré. Nous sommes polis et sensibles aux différences culturelles. Quittez le pays et tentez de trouver tout indicateur de vie intelligente émanant de cette grosse tache rose qui sépare les États-unis du Père Noël. Seules les catastrophes environnementales et les séparatistes québécois mettent le Canada sur la scène internationale.

Bien sûr, le Canada est tout simplement un secret bien gardé. Nous nous retrouvons constamment à la tête de la liste des pays qui offrent la meilleure

Département de médecine d'urgence, Université d'Ottawa, présentement en sabbatique à Harare, Zimbabwe qualité de vie au monde publiée annuellement par les Nations Unies. Même sans le bénéfice du CRTC, les stations de radio à travers le monde font tourner Céline Dion, Bryan Adams, Alanis Morissette et Shania Twain, qu'on croit évidemment Américains. Ce système de soins de santé impossible et mal financé que les médecins canadiens aiment détester fait l'envie du reste du monde.

Prenez votre journal américain de médecine d'urgence préféré et consultez la liste des collaborateurs. Pas mal de Canadiens, non? Nos sommités en médecine d'urgence méritent plus de reconnaissance que ce qui leur est accordé. Nous sommes la deuxième plus vieille spécialité de MU à avoir été établie au monde et l'un des quatre membres fondateurs de la Fédération internationale de médecine d'urgence. La contribution canadienne à la littérature mondiale en médecine d'urgence dépasse largement notre taille modeste.

Mais avons-nous vraiment besoin d'un autre journal de MU? En fait, parmi les 3330 journaux répertoriés dans Index Medicus, seulement 12 traitent de médecine d'urgence et deux de ceux-là sont publiés en allemand. Parmi les 10 journaux publiés en anglais, 3 proviennent du RU et 7 des États-Unis. Trois de ces journaux traitent spécifiquement des sous-spécialités comme la médecine de désastre et la médecine d'urgence pédiatrique.

Le système canadien de santé est unique en son genre et, comme nous le savons tous, la médecine d'urgence en est le moteur. La MU présente des défis génériques universels, mais les points forts, les problèmes et les solutions spécifiques à la MU canadienne sont nettement différents de ceux du reste du monde. Le financement de notre système est unique. Lire sur la menace de la maîtrise des dépenses de santé vous ennuie-t-il mortellement? Nous n'avons pas de COBRAs qui nous épient à l'urgence, mais nous avons nos propres démons, du genre «largage» et «pénurie de lits», pour n'en nommer que deux.

Tentez de convaincre un urgentologue américain que l'assurance de la qualité concerne l'amélioration des soins aux patients et non le contrôle des primes de malpractice. Et nous ne sommes pas seulement différents des Américains; essayez donc de discuter de la valeur de la certification CCMF(MU) avec un Australien ou un Écossais. Notre mélange multiculturel harmonieux de citoyens nous oblige rarement à effectuer des thoracotomies, mais nos voyages à travers le monde nous exposent à ramener dans nos bagages toutes les maladies et conditions qui existent sur la planète.

Les médecins d'urgence canadiens continueront de publier dans la presse internationale, contribuant à la vente de revues et de remèdes pour leurs commanditaires. Mais le moment est maintenant venu d'avoir le choix de publier au Canada et de partager l'expérience canadienne en MU avec le reste du monde. Il nous faut un journal canadien de médecine d'urgence. 